\title{
Surgery of the rheumatoid cervical spine
}

\author{
R A Johnston, I G Kelly
}

Patients with rheumatoid disease who are admitted to hospital will often show radiological evidence of the disease process in the cervical spine. ${ }^{1}$ The atlantoaxial level is most commonly affected with subaxial levels being less commonly involved. ${ }^{2}$ Only a minority of these patients will have neurological symptoms of this cervical disease, however.

\section{Pathology and patterns of disease}

The synovitis of rheumatoid disease can affect the facet joints, the neurocentral joints of Luschka, and the synovial joint between the odontoid peg and the transverse ligament of the atlas. It causes destruction of intervertebral discs and vertebral bodies and leads to ligamentous laxity. ${ }^{3}$ These erosive changes can be shown on plain radiographs as abnormal movement of vertebral bodies, and the clinical expression of this depends upon the level and degree of disease. The synovium can give rise to clinical problems in the absence of abnormal movement by acting as a space occupying lesion causing root or spinal cord compression. In 1951 Davis and Markley recorded cervical myelopathy in $16 \%$ of their cases who did not show any cervical subluxation. ${ }^{4}$

At the atlantoaxial level stretching and destruction of the transverse part of the cruciate ligament complex allows the atlas vertebra to move forward relative to the axis. This results in the spinal cord being compressed between the posterior arch of the atlas and the odontoid peg. Disease of the synovial joint between the odontoid process and the atlas vertebra causes an expansion of this joint, leading to anterior cord compression by the mass of pannus. In some cases the odontoid process is totally eroded by the inflammatory reaction. Most commonly the atlas moves forward relative to the axis, but rarely the atlas may move posteriorly if the distal portion of the odontoid process is completely eroded. Occasionally the anterior subluxation can become fixed.

The lateral masses of the atlas may collapse secondary to erosive changes in the atlantooccipital and atlantoaxial synovial joints. This leads to vertical subluxation of the odontoid process through the foramen magnum. The tip of the odontoid process, which may be expanded by surrounding pannus, is brought into contact with the cervicomedullary junction.

At subaxial levels anterior subluxation occurs as the result of facet joint disease and is most commonly seen at the $\mathrm{C} 5 / 6$ level, which is the level of greatest mobility. Any level may be affected, however, and it is common to see several levels showing anterior subluxation in a stepwise fashion. Subaxial involvement is considerably less common than atlantoaxial rheumatoid disease.

A prospective study by Pellicci et al went some way towards demonstrating the natural history of atlantoaxial and subaxial rheumatoid disease. ${ }^{5}$ Over a five year period they found that $80 \%$ of their patients showed radiological progression while neurological deterioration occurred in $36 \%$. Cervical pain showed a tendency to improve and only $13 \%$ of the patients who began with radiological evidence of disease came to surgical treatment. It seems likely that all seropositive patients will develop radiological changes in the cervical spine with the passage of time, but neurological deterioration is not inevitable. There is poor correlation between the degree of radiological disease of the cervical spine and the clinical neurological condition. Patients with relatively severe change on radiographs may be asymptomatic, and Pellicci reported that $50 \%$ of patients in his study who had severe subluxation were free from symptoms.

Vertical subluxation of the odontoid process is recognised as one of the most serious complications of rheumatoid disease and may affect up to $10 \%$ of patients who have cervical spine disease. ${ }^{67}$ Slatis noted progression of the vertical subluxation during a four year period in 12 patients who were treated conservatively, but also observed that the natural history of vertical subluxation is poorly understood, which makes evaluation of conservative and operative treatment difficult.

Not all patients with myelopathy show continued deterioration and some may be managed without surgery. Marks and Sharp, however, found that the use of a cervical collar for patients with a mild or non-progressive myelopathy resulted in seven deaths out of 15 patients within six months, though not all of these deaths were directly attributable to quadriparesis. ${ }^{8}$ Patients who underwent surgical cervical fusion fared better. Further evidence of the poor outcome associated with non-operative management of myelopathy was produced by Meijers et al. ${ }^{9}$

\section{Clinical features}

Rheumatoid compression of the spinal cord or nerve roots results in long tract signs and root pain. The commonest form of root pain is caused by anterior subluxation of the atlas upon the axis leading to suboccipital (C2 root) pain. This can be severe and is usually episodic, being 
provoked by sudden movement. It may occur in combination with a myelopathy, but more commonly occurs in isolation, although it may predate spinal cord compression. Posterior cervical pain or pain referred into the trapezius and interscapular region are not common with rheumatoid disease as they are in spondylitic degenerative disease.

It can be very difficult to distinguish between gait disturbance due to joint disease and that due to corticospinal tract compression. Spinal cord rheumatoid disease may give the patient a sensation of heaviness or fatigability in the legs. Often the patient has had many years of joint disease and she 'knows her joints', making it possible to distinguish between walking problems associated with joint inflammation and walking problems due to 'something else'. In the presence of cervical spine rheumatoid disease this 'something else' is spinal cord involvement. Hyperreflexia and extensor plantar responses, if present, confirm the clinical history. Paraesthesia, numbness, and brachialgia usually affect the arms, but sometimes these symptoms may be due to peripheral neuropathy associated with rheumatoid disease. The overall difficulty in diagnosing a myelopathy was noted by Marks and Sharp, who reported a mean delay of 31 weeks between the first neurological symptoms and the diagnosis of spinal cord disease. $^{8}$

\section{Radiological assessment}

Lateral projections of the cervical spine performed in flexion and extension (figs 1 and 2) are the most common examinations for showing abnormal movement at the $\mathrm{Cl}-2$ level. Conventional teaching says that a distance of greater than $3 \mathrm{~mm}$ between the anterior arch of atlas and the front of the odontoid process is abnormal as is a distance of $3.5 \mathrm{~mm}$ between the

Figure 1 Plain radiograph of the cervical spine in extension.

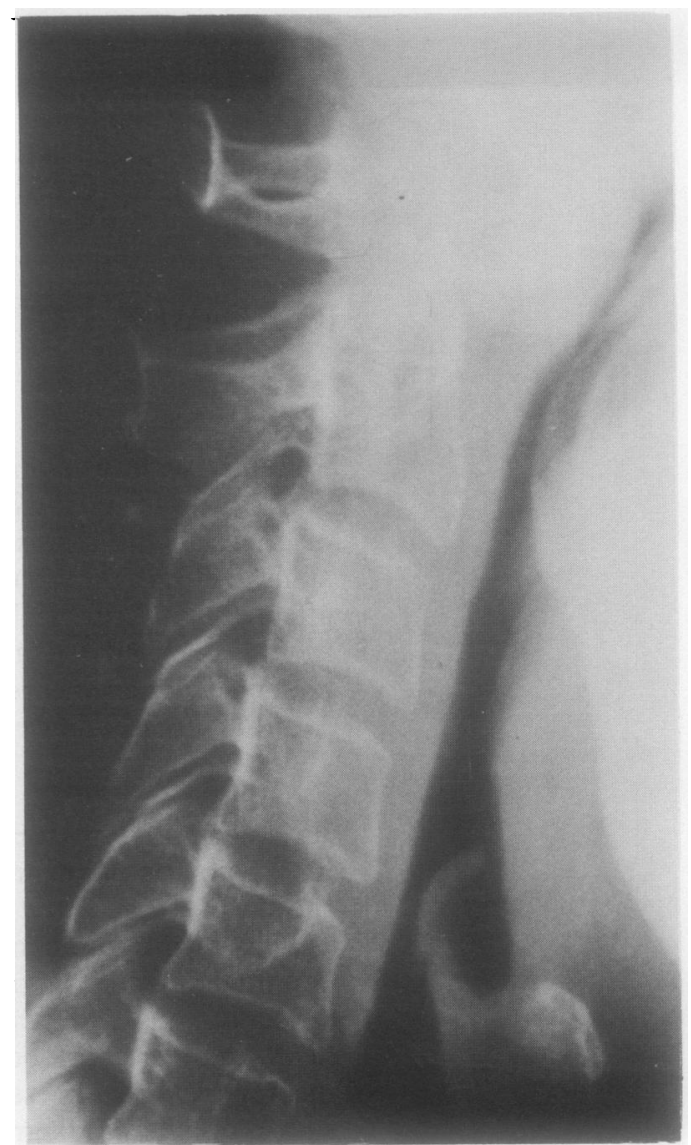

Figure 2. Plain radiograph of the cervical spine in flexion showing atlantoaxial subluxation.

posterior borders of adjacent subaxial vertebrae. This suggests that the available space for the spinal cord at the level concerned may be insufficient. The state of the odontoid peg and the lateral processes can be assessed by 'open mouth' views, though disease of the temporomandibular joint can make this difficult. Coexistence of vertical subluxation may conceal the amount of anteroposterior movement at the atlantoaxial level because the broader base of the odontoid peg comes to lie opposite the anterior arch of the atlas. The amount of vertical translocation can be calculated by several standard radiological measurements. ${ }^{10}$

Plain radiographs provide the clinician with a considerable amount of information about the degree of joint disease and bone position, but any conclusions about compression of the spinal cord or medulla are by implication only. Direct evidence of spinal cord compression is obtained with contrast enhancement or magnetic resonance imaging. Myelography has been largely superseded by computed tomography myelography (CTM) and magnetic resonance imaging (MRI). Both CTM and MRI should be carried out in flexion and extension positions (fig 3). Computed tomography myelography primarily shows bone tissue with the water soluble contrast surrounding the spinal cord. Horizontal sections through the craniocervical junction to the base of $\mathrm{C} 2$ are very helpful in indicating the amount of anteroposterior movement and also whether any rotation of the atlas is present. Sagittal images need to be reconstructed from these horizontal sections, whereas MRI is able to provide images 


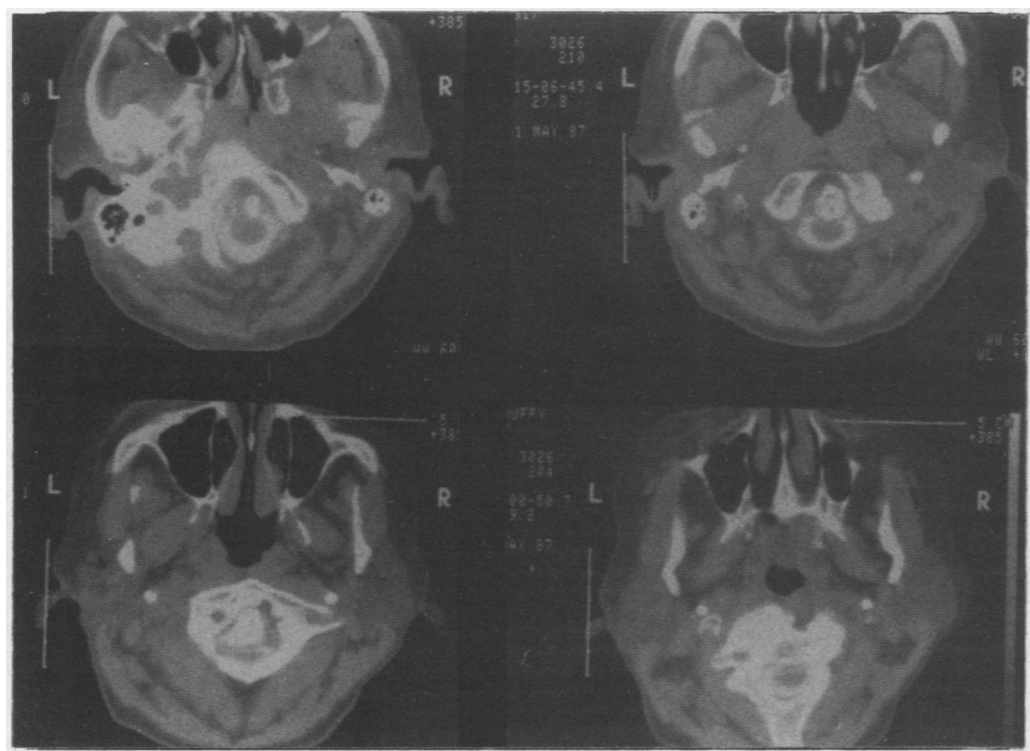

Figure 3 Computed tomography scan showing cord compression due to rheumatoid disease of the periodontoid synovial joint.

in almost any plane required, showing the spinal cord, medulla, inflammatory pannus, and fat pads. Magnetic resonance imaging has been shown to be as accurate as CTM in evaluation of most aspects of rheumatoid disease at the $\mathrm{Cl}-2$ level (figs 4 and 5)..$^{11}$

Direct imaging of neurological compression provides the basis for a fundamental change in surgical decision making. Santavirta et al indicated this when referring to myelography and CT, by stating that it is the compression of neural tissue rather than the size or amplitude of bony subluxation which is important. ${ }^{13}$ Magnetic resonance imaging permits repeat examination of patients who may be asymptomatic from their cervical disease, without the necessity for intrathecal contrast, and allows evaluation of the progression of their disease. The surgeon no longer has to assume that reduction of bony subluxation produces relief of neural compression. With good positional imaging reduction of

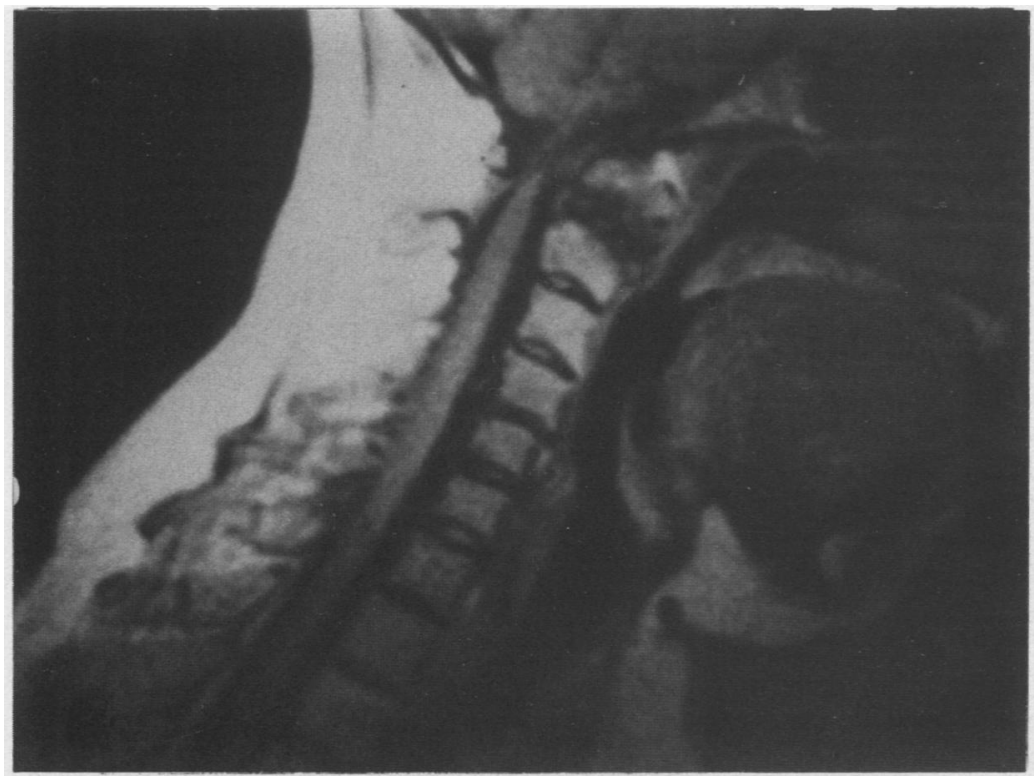

Figure 4 Magnetic resonance imaging scan (flexion) showing severe cord compression due to atlantoaxial subluxation. compression can be seen to occur (or not) with flexion or extension, and this will influence the type of surgery to be carried out.

\section{Indications for surgery}

The generally accepted indications for surgical intervention are neurological deterioration, which is progressive, and cervical/suboccipital pain which is not controlled by simple means. ${ }^{13-16} \mathrm{~A}$ more controversial indication is abnormal movement associated with compression indicated by MRI or CTM which is nevertheless asymptomatic. The question of prophylactic surgery is difficult to answer because of insufficient evidence about neurological and radiological progression. The balance of opinion at present suggests that surgery can safely be withheld until neurological features develop, though these can do so within a very short space of time. Patients can progress to severe quadriparesis within a matter of weeks and one of the problems in identifying this optimum window for surgical treatment is the well known difficulty of observing early signs of myelopathy by clinical examination. It is probably impracticable to follow up every patient with cervical rheumatoid disease by serial MRI examination, though it is by doing just this that we will be better able to correlate radiological progression and neurological deterioration.

\section{Operative treatment}

\section{ATLANTOAXIAL DISEASE}

In rheumatoid cervical spine disease the main problems are abnormal movement of vertebrae and accumulation of inflammatory tissue, both of which may lead to compression of the spinal cord or nerve roots. It follows that the principles of surgical treatment are $(a)$ to bring about decompression of the spinal cord or nerve roots, or both, and $(b)$ to restore structural integrity to the vertebral column. For most of the past 25 years posterior cervical fusion has been the most widely used operative procedure. This was advocated and described by Hamblen, ${ }^{16}$ Newman and Sweetnam, ${ }^{17}$ and even today it is probably the most commonly used surgical technique.

Ranawat et al found that the results of posterior surgical intervention were generally disappointing. ${ }^{10}$ Only $42 \%$ of their patients showed neurological improvement, while $10 \%$ showed evidence of continued deterioration. This was set against an early (less than four months) postoperative mortality of $27 \%$, with a further mortality of $18 \%$ up to two years after surgery. In the series reported by Zoma et al it was found that the operative success rate with posterior surgery was only $57 \%$, including some patients who underwent two procedures. ${ }^{14}$ Their early postoperative mortality was $8 \%$. Other reports also suggest that posterior surgery is not the complete answer to operative management of cervical rheumatoid disease. ${ }^{18}{ }^{19}$ This is not really surprising considering the various types and combinations of atlantoaxial subluxation and subaxial disease which are being treated by variations of one operation. 


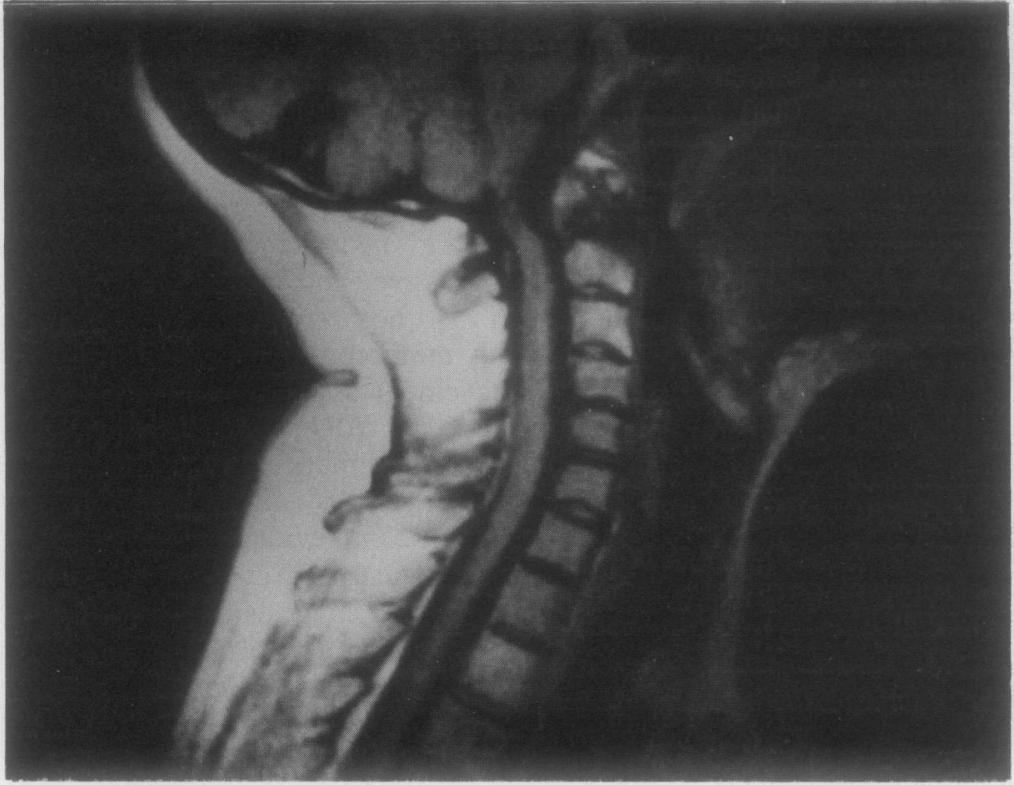

Figure 5 Magnetic resonance imaging scan (extension) showing that cord compression is still present even though some bony reduction has occurred. Soft tissue compression due to pannus.

The anterior approach to the clivus-atlasodontoid region was reported early this century. ${ }^{20}$ Until fairly recently it was recognised as a hazardous approach both for the surgeon and the patient because of the risk of meningitis, wound breakdown, cerebrospinal fluid fistula, poor access, and probably also because of inadequate instrumentation. More recently, the operation has been revived and modern technical improvements have responded to these earlier problems, making this a safe procedure. ${ }^{21-23}$ Direct decompression of the cervicomedullary region by removal of the odontoid process and associated pannus is a logical approach to ventral compression of the spinal cord.

The re-emergence of transoral approaches to the odontoid region has added a new dimension to surgical management of cervical rheumatoid disease at the atlantoaxial region. In the most common situation where there is anterior subluxation of $\mathrm{Cl}$ on $\mathrm{C} 2$, and with the less common vertical subluxation, decompression of the spinal cord/medulla can be achieved by removing the odontoid peg and the surrounding pannus. Where there is vertical subluxation it is also necessary to remove the lower one third or more of the clivus. The operation is carried out through the contaminated field of the mouth and nasopharynx, but local infections are very uncommon. This may be attributed to the use of prophylactic antibiotics, but perhaps is more likely to be due to the good healing properties of the well vascularised tissues in this region. A vertical incision is made in the posterior pharyngeal wall through the constrictor muscles and this brings the surgeon almost immediately to the anterior arch of atlas. Increased access in a cranial direction is achieved by retraction or splitting of the soft palate. With advanced cases of vertical subluxation it is necessary to open the hard palate, though the surgeon may use an alternative approach through a maxillotomy. The odontoid process, or what remains of it, is removed completely, making sure that the tip end is severed from any remaining ligamentous attachments. It is often possible to observe the remains of the very lax transverse ligament. Pannus is removed as completely as possible to complete the decompression down to the membrana tectora.

When the anterior decompression has been achieved it is usual to carry out a posterior stabilisation procedure under the same anaesthetic. This makes for a longer operative procedure, but the alternative of a two stage procedure implies an interim period of cervical traction, which will delay recovery and rehabilitation. The posterior surgical approach entails exposure of the suboccipital region to the mid or lower cervical level. Exploration of the arch of $\mathrm{Cl}$ will confirm any radiological diagnosis of posterior cord compression and if necessary a wide $\mathrm{Cl}$ laminectomy is carried out. When the lateral masses are intact the surgeon may choose between an occipital/C1/C2 or a $\mathrm{Cl} / \mathrm{C} 2$ fixation/ fusion procedure. With vertical subluxation the lateral masses of the atlas are eroded and an occipitocervical procedure is necessary. A number of different methods may be used, including a combination of wire, bone graft, and acrylic cement ${ }^{24}$ (fig 6 ) or the technique of sublaminar wiring with a Ransford loop (fig 7). ${ }^{24}$ Both methods are satisfactory and provide immediate internal stability to the cervical spine. Both methods entail occipitocervical fixation/fusion. Use of wire and bone graft alone (fig 8) will necessitate the use of an external orthosis until bone fusion has occurred. The advantage of the former two methods in providing immediate internal stability is that rehabilitation may begin on recovery from the operation.

The evidence from current publications and from our own experience indicates that this combined operation is well tolerated by patients, who are often elderly and frail women. Considering the serious nature of the condition, the

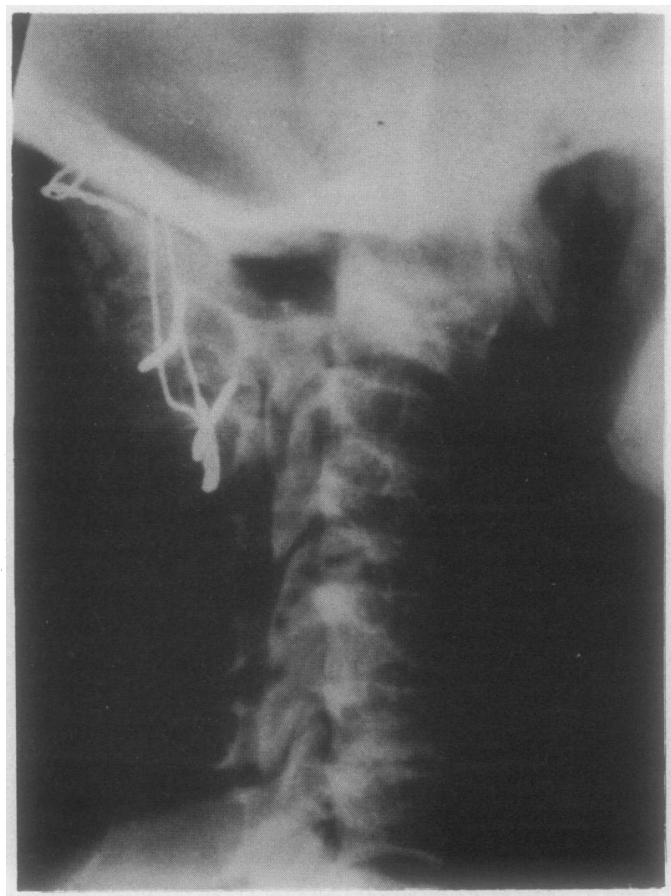

Figure 6 Brattstrom occipito-C2 fixation and fusion. 


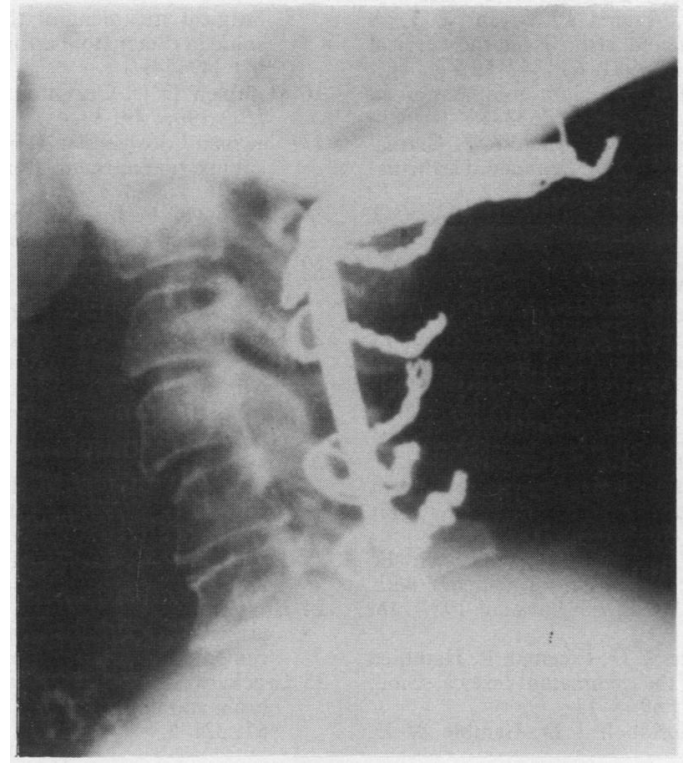

Figure 7 Occipitocervical fixation by Ransford loop.

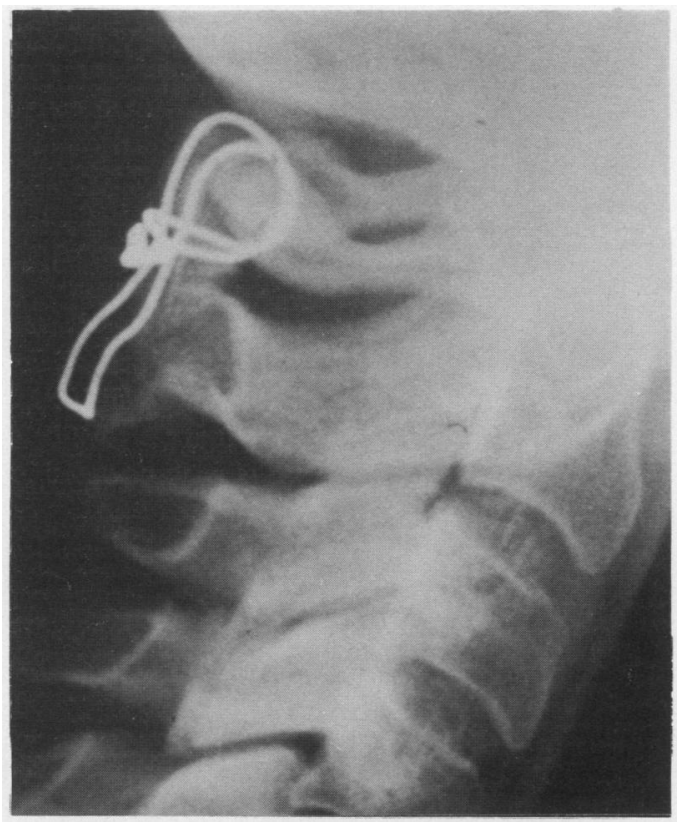

Figure 8 C1-2 wiring with bone graft.

general state of health of these patients, and the major surgical endeavours needed to correct the problem, there is an acceptably low morbidity and mortality. Hadley reported two deaths within nine weeks of surgery in 21 patients. ${ }^{23}$ Most of these patients, however, had only the anterior procedure performed. We have found a similar mortality. Complications such as pharyngeal wound breakdown, cerebrospinal fluid fistula, and infection do occur, but are uncommon.

Neurological improvement was reported in $90 \%$ of surviving patients (mixed pathology, including rheumatoid disease) by Hadley, ${ }^{23}$ though little detail of the improvement was given. In his early series Crockard found neurological improvement in 15 of 17 patients, and this rate of improvement has been maintained (personal communication). These very encouraging results require more detailed neurological verification to establish the true position of transoral decompression surgery for atlantoaxial disease.

\section{SUBAXIAL DISEASE}

The surgical treatment of subaxial rheumatoid disease is perhaps less clearly defined. To date the most common treatment is posterior fixation/fusion, ${ }^{10}$ though this may not actually achieve any significant cord decompression. Anterior cervical decompression alone seems to be insufficient to stabilise the cervical spine and a combined anterior and posterior operation has been advocated. ${ }^{25}$ At present there is probably insufficient evidence to indicate firmly one form of surgery in preference to another.

\section{Summary of treatment}

In Glasgow we advocate operative treatment for patients who have rheumatoid cervical spine disease with neurological deterioration, either as a myelopathy or radiculopathy, which includes intractable suboccipital (C2) pain. Although occasionally noting considerable radiological changes in patients without neurological features, we have not as yet taken up the option of prophylactic cervical surgery. This awaits further information on the natural history and progress of the disease; accumulation of serial CT and MRI data should help in this respect. Before the days of CT and MRI the term 'reduction' referred to bony alignment. Reduction should now refer to neurological compression. When there is cord compression due to atlantoaxial disease, and this does not reduce by extending the cervical spine, we prefer to use a combined transoral decompression and posterior occipitocervical fusion procedure. When the neural decompression does reduce (usually with extension) then a posterior fusion procedure at $\mathrm{Cl}-2$ is used. For vertical subluxation we use the combined anterior and posterior operation as it is virtually impossible to achieve any bony reduction, and thereby any neurological improvement, by traction. We feel that simply holding the spine in the abnormal position is insufficient. Subaxial disease is at present treated by anterior decompression and fusion using a modified Cloward technique combined with posterior fixation.

Technical advances continue to increase the repertoire of surgical procedures available, but we must not lose sight of the importance of decision making before the operation based on sound information about the natural history of the disease in the cervical spine.

1 Swann M. Surgical treatment of cervical spine in rheumatoid arthritis. Ann Acad Med Singapore 1983; 12: 233-42.

2 Ornilla E, Ansell B M, Swannell A J. Cervical spine involvement in patients with chronic arthritis undergoing orthopaedic surgery. Ann Rhewm Dis 1972; 31: 364-8.

3 Martel W D. Pathogenesis of cervical discovertebral destruction in rheumatoid arthritis. Arthritis Rheum 1977; 20: tion in rit-25.

4 Davis F W, Markley H E. Rheumatoid arthritis with death from medullary compression. Ann Intern Med 1951; 35: 451-61. 
5 Pellicci P M, Ranawat C S, Tsairis P, Bryan W J. A prospective study of rheumatoid arthritis of the cervica spine. $\mathcal{F}$ Bone foint Surg [Am] 1981; 63: 342-50.

6 Davidson $R$ C, Herndon J H. Brain stem compression in rheumatoid arthritis. FAMA 1977; 238: 2633-4.

7 Slatis P, Santavirta S, Sandelin J, Konttinen Y T. Cranial subluxation of the odontoid process in rheumatoid arthritis. 7 Bone foint Surg [Am] 1989; 71: 189-95.

8 Marks J S, Sharp J. Rheumatoid cervical myelopathy. $Q \mathcal{F}$ Med 1981; 199: 307-19.

9 Meijers K A E, Cats A, Kvemer H P H, Lugendii $K$ W, Onrlee G J, Thomeer $R T$ W M. Cervical myelopathy in Onrlee G J, Thomeer R T W M. Cervical myelopathy in

10 Ranawat C S, O'Leary P, Pellicci P, Tsairis P, Marchisello P Dorr $L$. Cervical spine fusion in rheumatoid arthritis. $\mathscr{f}$ Bone foint Surg [Am] 1979; 61: 1003-10.

11 Bundschuh C V, Modic M T, Kearny F, Morris R, Deal C. Rheumatoid arthritis of the cervical spine: surface coil $M R$ imaging. AfNR 1988; 9: 556-71.

12 Beltran J, Caudill J L, Herman L A, et al. Rheumatoid arthritis: MR imaging manifestations. Radiology 1987; 165: 153-7.

13 Santavirta S, Kankaanpaa V, Sandelin J, Laasonen E, Konttinen Y T, Slatis P. Evaluation of patients with rheumatoid cervical spine. Scand $\mathcal{f}$ Rheumatol 1987; 16: 9-16.

14 Zoma A, Sturrock R D, Fisher W D, Freeman P, Hamblen $\mathrm{D}$ L. Surgical stabilisation of the rheumatoid cervical spine. f Bone foint Surg [Br] 1987; 69: 8-12.

15 Ferlic D C, Clayton M L, Leidholt J D, Gamble W E.
Surgical treatment of the symptomatic unstable cervical pine in rheumatoid arthritis. $\mathcal{F}$ Bone foint Surg [Am] 1975; 57: $349-54$.

16 Hamblen D L. Occipito-cervical fusion. 7 Bone foint Surg [Br] 1967; 49: 33-45.

17 Newman P, Sweetnam D R. Posterior fusion for atlanto-axia subluxation in rheumatoid arthritis. $\mathcal{F}$ Bone foint Surg $[\mathrm{Br}$ 1969; 51: 423-8.

18 Cregan J C F. Internal fixation of the unstable rheumatoid cervical spine. Ann Rheum Dis 1966; 25: 242-52.

19 Crellin R Q, MacCabe J J, Hamilton E B D. Severe subluxation of the cervical spine in rheumatoid arthritis. f Bone foint Surg [Br] 1970; 52: 244-51.

20 Kanavel A B. Bullet located between the atlas and the base of the skull: technic of removal through the mouth. Surgical Clinics of Chicago 1917; 1: 361-6.

21 Apuzzo M L J, Weiss M H, Heiden J S. Transoral exposure of the atlanto-axial region. Neurosurgery 1978; 3: 201-7.

22 Crockard H A, Essigman W K, Stevens J M, Pozo J L Ransford A O, Kendall B E. Surgical treatment of cervical cord compression in rheumatoid arthritis. Ann Rheum Dis 1985; 44: 809-16.

23 Hadley M N, Spetzler R F, Sonntag V K H. The transora approach to the cervical spine. F Neurosurg 1989; 71: 16-23.

24 Brattstrom $\mathrm{H}$, Granholm L. Atlanto-axial fusion in rheumatoid arthritis. A new method of fixation with wire and bone cement. Acta Orthop Scand 1976; 47: 619-28.

25 Crockard H A. The transoral approach to the base of the brain and upper cervical cord. Ann R Coll Surg Engl 1985; 67: $321-5$. 